Orbis Tertius, vol. XXIII, nº 27, e067, junio 2018. ISSN 1851-7811

Universidad Nacional de La Plata

Facultad de Humanidades y Ciencias de la Educación

Centro de Estudios de Teoría y Crítica Literaria

\title{
La Crónica X: sus interpretaciones y propuestas
}

\section{Clementina Battcock * \\ * Instituto Nacional de Antropología e Historia, México.}

Cita sugerida: Battcock, C. (2018). La Crónica X: sus interpretaciones y propuestas. Orbis Tertius, 23 (27), e067. https://doi.org/10.24215/18517811e067

cc)(-) Esta obra está bajo licencia Creative Commons Atribución-NoComercial-CompartirIgual 4.0 Internacional http://creativecommons.org/licenses/by-nc-sa/4.0/deed.es_AR 


\title{
La Crónica X: sus interpretaciones y propuestas ${ }^{1}$
}

\author{
Clementina Battcock \\ Instituto Nacional de Antropología e Historia, México
}

\section{ReSUMEN:}

En 1945 Robert Barlow postuló la existencia de un texto, denominado por él como "Crónica X", del que derivaron una multiplicidad de fuentes con similitudes estructurales que dan cuenta del pasado prehispánico de la Cuenca de México. Han sido varios los investigadores que se han dedicado a discutir e incorporar nuevos elementos a su propuesta original. El objetivo de este artículo es presentar un análisis que integre los argumentos de los estudios realizados para proponer líneas de investigación pendientes en torno a este debate historiográfico

Palabras Clave: Crónica, Memoria, Escritura, Indígena, Nahua.

\section{Abstract:}

In 1945 Robert Barlow postulated the existence of one text, named by him as "Crónica X", which was supposed to be the common source of a lot of documents with structural similarities about the prehispanic past of Mexican people. Many researchers have analyzed this document and have also incorporated new elements to Barlow's original postulates. The objective of this article is to present an analysis that integrates the main points of those researchers as well as to offer a new perspective in this historiographical debate.

KeYwords: Historiography, Chronicle, Prehispanic, Mexico, Research.

La conferencia titulada "La 'Crónica X': versiones coloniales de la historia de los mexica tenochca" fue dictada por Robert Barlow en 1945. Este estudio sentó las bases para establecer la vinculación estructural de cinco fuentes: el Manuscrito Tovar, el libro VII de la Historia natural y moral de las Indias de José de Acosta, el Códice Ramírez, el volumen correspondiente a historia de la Historia de las Indias de Nueva España e Islas de Tierra Firme de fray Diego Durán, y la Crónica mexicana de Hernando Alvarado Tezozomoc. ${ }^{2}$ En las siete décadas transcurridas desde entonces, diversos especialistas mexicanos y extranjeros han hecho sus propios planteamientos y aportaciones a esa idea seminal y los han difundido en libros, artículos y tesis doctorales. Sin embargo, nadie se ha ocupado de reunir todas estas adendas para ofrecer un panorama general del estado de la cuestión. En este breve trabajo realizo un repaso de los diferentes estudios y planteamientos que, a partir de la propuesta pionera de Barlow, se han realizado hasta nuestros días. Asimismo, propongo algunas líneas de investigación futura que puedan contribuir a la tarea colectiva de esclarecer lo que todavía constituye uno de los mayores problemas heurísticos y hermenéuticos de la historiografía novohispana de tradición indígena. ${ }^{3}$

\section{SUS ESTUDIOS, ENFOQUES Y PROBLEMÁTICAS}

El inicio de la polémica sobre la denominada Crónica $X$ se remonta al siglo XIX, con las conjeturas de José Fernando Ramírez acerca de la posible existencia de una fuente en náhuatl -posteriormente traducida al castellano- que sirvió como base para la realización de diversas obras de tradición mexica, entre las que se encontraba la de Hernando Alvarado Tezozomoc (1987: 9-15). La idea de Ramírez fue bien recibida por los estudiosos de la época, entre los que se encontraba Alfredo Chavero, quien elaboró un estudio comparativo entre las diferentes obras citadas y concluyó que las cuatro crónicas, el Códice Ramírez, Durán, Acosta y 
Tezozomoc son en realidad una sola, y esta es la única fuente verdadera para escribir la historia del poderoso imperio tenochca (1987: 161-167).

Para 1945 ya se habían identificado cinco fuentes con evidentes similitudes estructurales: el Manuscrito Tovar, el libro VII de la Historia natural y moral de las Indias de José de Acosta, el Códice Ramírez, el volumen correspondiente a historia de la Historia de las Indias de Nueva España e Islas de Tierra Firme de fray Diego Durán y la Crónica mexicana de Hernando Alvarado Tezozomoc. Según el dictamen de Robert Barlow, las tres primeras no serían sino variantes de una versión corta de la obra de Durán, con lo que el problema se reducía a dar con la fuente única a partir de la que se habrían originado las obras de Durán y Tezozomoc. A esta obra perdida Barlow la denominó Crónica $X^{4}$ y consideró que debe haber sido escrita en lengua náhuatl, por un indígena, entre los años de 1536 y 1539, y que iba acompañada de dibujos (1990: 20-27).

En 1947, Ignacio Bernal publicó algunas observaciones sobre la Crónica $X$ en un pequeño y acotado artículo, en el que argumentó que los datos calendáricos no eran suficientes para sustentar, por un lado, la idea de que la Crónica X y el Manuscrito Tovar eran lo mismo y, por otro, la fecha de elaboración de la Crónica $X$ (1947: 125-134). Para corroborar su teoría, reconstruyó el calendario a partir del Códice Ramírez y de la Historia de las Indias de Durán, y llegó a la conclusión de que las fechas basadas en el calendario eran inválidas, por lo que descartó la fecha que propuso Barlow de 1536-1539 para la elaboración de la Crónica X y dejó abierta la discusión a futuras investigaciones. En 1964, Ignacio Bernal desarrolló anotaciones en torno a la Crónica $X$ dentro de la publicación de la Historia de las Indias, editada por Doris Heyden, donde, además de brindar un contexto amplio de la obra de fray Diego Durán, Bernal avaló la idea de una relación directa entre la Historia de Durán, el Códice Ramirez, Tovar y el capítulo 7 de Acosta. Sin embargo, puntualizó que las historias de Tezozomoc y de Durán son obras parecidas pero no idénticas, y que dicha semejanza se debe a que los dos autores utilizaron el Manuscrito Tovar. Aclarado el punto anterior, Ignacio Bernal explicó que la Crónica $X$ pudo haber existido de forma independiente a las obras de Tovar, Durán y Tezozomoc. Es decir, que el jesuita Tovar habría tomado información de varios manuscritos pintados y narraciones escritas, a los cuales habría organizado en una historia de eventos ordenados consecutivamente. Tovar se valió de informantes indígenas, por lo que se puede concluir que su trabajo no estuvo basado en la Crónica $X$ y que, en el mejor de los casos, sólo la utilizó como una de sus fuentes. Por último, Bernal opinó que los manuscritos pintados y las narrativas escritas mencionadas por Tovar, y utilizados en su primera historia, podrían haber sido parte del material reunido por el franciscano Andrés de Olmos, aunque aclara que para probarlo se necesitaría un estudio comparativo.

Más tarde, en 1951, Paul Kirchhoff formalizó una perspicaz observación sobre la Crónica mexicáyotl ${ }^{5}$ (1951:225-227), donde indicó que la segunda parte de esta obra fue escrita por el cronista chalca Chimalpain y no por Alvarado Tezozomoc, ya que el estilo y la forma de escribir, así como la información cronológica, coinciden con la Séptima Relación de Chimalpain (1951: 227). A ello agregó que, en la segunda parte de la Crónica mexicáyotl también se dejan ver correcciones realizadas por éste; por lo que todo esto en conjunto muestra el conflicto que hay cuando un autor al escribir se encuentra entre dos tradiciones.

Stephen Colston realizó en 1973 un análisis historiográfico de la Historia del dominico fray Diego Durán y planteó como primera observación acerca de la Crónica $X$ y su ubicación que quizá formara parte de la colección de manuscritos ordenados por el virrey Martín Enríquez y, en consecuencia, habría sido utilizada por el padre Juan de Tovar para escribir su primera historia. A partir de esta idea se inclina por la posibilidad de que el denominado Tovar I no fuera una traducción literal de la Crónica X y que sólo se ocuparan ciertas partes del escrito. El estudio de Colston concluyó que la famosa Crónica $X$ no fue una sino varias Crónicas $X$ estrechamente relacionadas y originadas a partir de la tradición oral, o que quizá fueron papeles y libros coleccionados y obtenidos de bibliotecas indígenas antiguas. Este mismo investigador publicó en 1977 sus indagaciones referentes a la fecha de elaboración de la Crónica $X$. Su hipótesis partió de la fecha propuesta en 1945 (entre 1536 y 1539), y refutó este supuesto porque encontró suficientes variaciones textuales entre las referencias de Durán y Tezozomoc para suponer que habría existido una fuente textual común. Además 
propuso que no existió una fuente única, sino que ambos cronistas se basaron en la tradición oral de la rama tenochca (por las referencias que se realizan en torno a Cihuacóatl y Tlacaélel), con la diferencia de que Durán dependió de una fuente escrita y Tezozomoc de una versión oral directa. Por otra parte, Colston señaló que la Crónica $X$ fue escrita en náhuatl, con caracteres latinos, redactada después de la Conquista y terminada para la fecha en que Durán la usó como referencia, es decir, entre 1579 y 1581 . También analizó brevemente la referencia que hizo Durán al autor de la Historia Mexicana al describirlo como "un indio", y dedujo de ello que el texto debe haber sido escrito varios años después de la Conquista, cuando un indio, joven o maduro, ya había estado inmerso en la educación española. Así, la Crónica $X$ debe haber sido redactada después de 1531 y antes de 1579-1581, aunque advirtió que faltan evidencias que confirmen la existencia de tal obra.

La tesis doctoral de Sallie Elizabeth Brennan (1988), presentada en la Universidad de Rochester y publicada en 1988, fue una obra que se dedicó al examen del tiempo, espacio, objetivos y técnicas de narrativa histórica presentes en la Crónica mexicáyotl. Una sección de su estudio se detuvo en el análisis de las fechas de los gobernantes tenochcas propuesta por Kirchhoff, donde Brennan se percató de que parte de esta reconstrucción se basó en documentos que tienen un origen común: la Crónica X. La investigadora explicó que los datos pueden localizarse en la Crónica Mexicana, el Códice Ramírez, la historia Durán y la Séptima relación de Chimalpain, quien a su vez los plasma en la Crónica mexicáyotl. Brennan analizó la lista y aseveró que quizás la Crónica $X$ pudo haber sido de tradición tlatelolca y que la cronología que propuso Kirchhoff es errónea, ya que la cronología no corresponde a la Crónica $X$, pues la Crónica mexicana no proporciona fechas prehispánicas o cristianas asociadas con las fechas de gobierno o muerte de los gobernantes. Asimismo, la investigadora hizo notar que Tezozomoc y su Crónica Mexicana y su Crónica mexicáyotl son lo suficientemente semejantes como para constituir una sola tradición, aunque se debe reconocer que existen diferencias entre ellas y la información contenida en la Crónica mexicana es un relato intermedio entre la Historia de las indias de Durán y la Crónica mexicáyotl. Por último, afirmó que la Historia de los mexicanos por sus pinturas combina más capítulos encontrados en la Crónica mexicáyotl y los Anales de Tlatelolco que cualquier otra fuente.

En 1998, el investigador Rafael Tena realizó un estudio cronológico e historiográfico relativo a la Crónica $X$,centrando su atención en el Códice Tovar y la Historia de Durán, en contraste con la tendencia general de ubicar el foco de la investigación en el texto de Tezozomoc. Tras el análisis profundo de ambos documentos, realizó una propuesta sobre la Crónica X: para escribir su primera Historia, Tovar se basó exclusivamente en las explicaciones verbales que le dieron los indígenas sobre una serie de códices entre 1575 y 1576 y no antes, además de haber sido reunidos por el virrey Enríquez. Por otra parte, a partir de 1578, para escribir su Historia, Durán ocupó un documento escrito en náhuatl, cuyo origen y fecha de composición no es posible precisar, pero que coincidía con la información de los informantes de Tovar, por lo que probablemente Tovar habría utilizado la Historia de Durán para escribir su segunda Historia. Por los elementos anteriores, la primera Historia de Tovar y la Historia de Durán derivan de explicaciones verbales y de un documento escrito, pues a pesar de ser independientes en su elaboración, resultan coincidentes en su contenido porque reflejan una misma tradición. Tena caracterizó esta tradición oficial como una "glosa" que afloraba en forma de comentario espontáneo en presencia de las pinturas antiguas y que en más de una ocasión pudo haber sido puesta por escrito. En cuanto al texto de Tezozomoc, posiblemente se apoyó en una relación escrita en náhuatl, idéntica o parecida a la utilizada por Durán, o que haya surgido directamente en la tradición oral, como hizo Tovar para componer su primera Historia. De esta tradición oral primigenia, cristalizada como glosa, derivaron en su momento, en el corto o mediano plazo, la primera Historia de Tovar, la Historia mexicana de Durán y la Crónica Mexicana de Tezozomoc, siendo estos los documentos más cercanos a la Crónica X(Tena 1997: 163-178).

Por su parte, Rocío Cortés (1998), en su tesis doctoral, al comparar y analizar el contenido de las obras, desmenuzó las narrativas y el discurso empleado por Hernando Alvarado Tezozomoc en la Crónica Mexicana y la Crónica mexicáyotl. En su análisis retomó el planteamiento de que la Crónica mexicana posiblemente 
fuera una traducción de la ya mencionada Crónica $X$ y que, al no tener noticia de ella podría pensarse que la primera obra de Tezozomoc es la traducción de varios textos orales y escritos, que también conoció Durán (1998: 123-127). A partir de esa afirmación y de la posible existencia de la Crónica X, Rocío Cortés consideró la omisión de ciertos pasajes en la transcripción de la Crónica Mexicana y la inclusión de los mismos en la Crónica mexicáyotl, y sostuvo que estas diferencias se debían a que la primera obra, por su temática y formato, iba dirigida a una audiencia europea, en tanto empleó la traducción como una estrategia discursiva; mientras que la segunda obra fue pensada para los mexica, por lo que utilizó un discurso legal y notarial, además de legitimar su antiguo linaje.

También en 1998, Ann Marie Graham presentó en su tesis de doctorado el mismo enfoque comparativo y abordó las influencias literarias e interpretativas de dos obras emparentadas con la Crónica X: la Crónica Mexicana de Tezozomoc y la Historia de las Indias de Fray Diego Durán. En su examen literario consideró el origen de la Crónica $X$ y sugirió que ésta sería producto de las indagaciones de los españoles sobre la vida de los indígenas a partir de un intercambio de documentos y fuentes que existían en el siglo XVI, y por tanto que serían estos hechos los que darían cuenta de la resultante diversidad de textos (Graham 1998). Bajo esta premisa, La Crónica $X$ sería un crisol en el que se habrían mezclado dos conciencias históricas distintas así como dos maneras de recordar la historia, y con toda seguridad habría sido escrita en náhuatl y estaría destinada a los españoles (Graham 1998: 12-19). Graham afirmó que la estructura de Tezozomoc y Durán hace posible la idea de que la Crónica $X$ haya tenido como objetivo reunir varios géneros históricos nahuas en un texto escrito al estilo europeo, y que incluso pudo ser una fuente pictográfica ya organizada y anotada. Esta propuesta dejó abierta la discusión acerca de la existencia de dos versiones de la misma (Graham 1998: 19).

Con el ánimo de profundizar en tal estudio, en 2003 José Rubén Romero Galván preparó un cotejo y análisis de las obras de Durán y Tezozomoc, mediante el cual corroboró su estrecha relación y su origen común. Sin haber podido encontrar en la Crónica mexicana ninguna referencia o indicio de que Hernando Alvarado Tezozomoc se hubiera basado en una historia prexistente, sí consideró evidente que el autor tradujo una relación originalmente escrita en lengua náhuatl (2003: 191). Para Romero Galván, la "relación original" o Crónica $X$ fue un documento cuya base eran códices de contenido histórico, y que incluyó información de tradición oral proveniente de un acervo que poseyó el virrey Martín Enríquez, que aprovechó el jesuita Tovar y que desapareció poco después. También adujo que es riesgoso afirmar que este documento contuvo dibujos, aunque sí la atribuyó a un autor indígena, descendiente mexica, conocedor de códices, de la lengua náhuatl, del abecedario latino, formado en el Colegio de Santa Cruz de Tlatelolco y que, acaso, haya respondido al nombre de Hernando Alvarado Tezozomoc, si no es que se trató de alguien muy próximo a este autor (Romero Galván, 2003: 192-195). Finalmente, data su factura en fecha posterior a 1576 y previa a 1580.

Dos años después del trabajo de Romero Galván, en 2007, la investigadora belga Sylvie Peperstraete puso su grano de arena en el montículo de análisis sobre la Crónica $X$ con su tesis de doctorado. Sus objetos de investigación fueron las láminas de la primera obra. Peperstraete planteó la posibilidad de que la Crónica mexicana haya seguido más de cerca la secuencia narrativa de la Crónica X (Kenrick Kruell 2013c: 321). Para ella, la crónica perdida debe haber sido una historia ilustrada, escrita alrededor de 1547 y 1560, y su autor un descendiente del linaje de Tlacaelel, a juzgar por la importancia que le confiere a tal personaje (Kenrick Kruell 2013c: 323). En un artículo de 2009, Peperstraete puso de relieve la utilidad inmediata de la reconstitución de los contenidos desaparecidos de la Crónica $X$, extraídos de una cuidadosa comparación de las obras de Diego Durán y Hernando Alvarado Tezozomoc. Así, señaló que en primer lugar tenemos una mejor comprensión de lo que debe ser similar al documento original, su contenido, su fecha de escritura y su autor. En este artículo, los mexicanistas también tienen a su disposición una base fiable para su trabajo, lo que permite un fácil manejo y uso crítico de los contenidos de tal crónica, pues la relación de ésta con otros documentos, algunos utilizados con frecuencia por los especialistas, podría definirse con precisión. Este último punto es particularmente instructivo para la información que proporciona y las preguntas que se plantean sobre la profesión histórica y la manera de escribir la historia de los siglos XVI y XVII. 
En 2008, María Castañeda publicó un artículo que parte de la supuesta existencia de una fuente pictográfica nombrada Códice $X$, que sirvió de base para otros documentos. Así, propuso que Tezozomoc, Chimalpain y Juan de Torquemada, además de la Crónica $X$, también utilizaron el corpus del Códice $X$ para escribir sus obras, con lo que se explica la semejanza que tienen en la forma de escribir y en el contenido de sus textos, y se inclina a pensar que se copiaron entre ellos. Al respecto, Castañeda aclaró que "copiar" era una práctica común en el siglo XVI, que no impide conocer las diversas fuentes consultadas por los autores y cómo relacionaron las fuentes pictográficas con las alfabéticas. En particular, consideró a la Crónica X como un documento conocido que circulaba en el siglo XVI, aunque que estaba resguardada por los frailes, y que el único que tuvo acceso a ella habría sido Tezozomoc, quien la habría copiado e insertado algunas partes de ella en la Crónica mexicáyotl (2008: 197). Dos años más tarde, en coautoría con Michel Oudijk, María Castañeda sacó a la luz un interesante legajo ubicado en el Archivo General de la Nación de México, que forma parte de un alegato de tierras entre Tlatelolco y el Pueblo de Guadalupe (Oudijk y Castañeda 2009:255-278). A juicio de los autores, el documento que presentaron las autoridades de Tlatelolco deja ver el uso de dos importantes documentos: la Crónica X y la Ordenanza del Señor Cuaubtémoc. La transcripción del texto posiblemente es el resumen de varios papeles en náhuatl y, al traducirlos, da la impresión de que podría ser la Crónica $X$, pues se trataría de un documento mixto, es decir que alterna pictografía y texto alfabético en nahuatl. Otra cuestión que apoya esta idea es que ninguno de los textos en manos de los otros cronistas fue publicado antes del siglo XIX, a excepción del texto del padre José de Acosta, impreso en 1590. Por ende, se puede deducir que es muy probable que el pueblo de Tlatelolco haya podido copiar la historia de la Crónica $X$ de uno de estos cronistas. Castañeda y Oudijk concluyen que la comparación del texto con las tres versiones de la Crónica $X$ permite explicar que el alegato de Tlatelolco está basado en un texto en náhuatl que parece ser el mismo que aquel en el que se basaron Juan de Tovar y José de Acosta, esto a través de constantes paralelismos en los textos. De tal manera podría suponerse que el texto en náhuatl encontrado entre los papeles utilizados en el juicio, y que era la fuente de origen de la primera parte del alegato de Tlatelolco, es la Crónica $X$, ya que en el siglo XVIII Tlatelolco todavía guardaba muchos documentos coloniales tempranos, entre ellos la Crónica $X$ y los Anales de Tlatelolco, u otra versión de los mismos.

En el 2010, Sylvie Pepertraeste propuso una nueva forma de analizar la Crónica mexicáyotl que tomó como eje principal la relación existente entre las diversas fuentes escritas. Para la autora, la Crónica mexicáyotl fue producto de dos escritores, Tezozomoc y Chimalpain, y fue elaborada a partir de la reunión de varias fuentes o extractos de fuentes, entre ellas algunos pasajes de la Crónica X (2010:7-37). Fue Tezozomoc quien escribió un texto original, copiado y modificado por Chimalpain, hecho en el que se basa la investigadora para identificar las diferentes fuentes utilizadas para su escritura. Peperstraete aclaró que Tezozomoc, como la mayoría de los autores de su época, no sólo juntó copias exactas de las fuentes principales, sino que también añadió detalles de fuentes adicionales y descripciones improvisadas de los dibujos que se encontraban en los manuscritos pictográficos, con el fin de formar una narrativa continua. De esta forma, el análisis de Pepertraeste se centra en la identificación de las diferentes fuentes que utilizaron los autores, para así comprender mejor la elaboración de la Crónica mexicáyotl. La autora elaboró un cuadro con las fuentes mencionadas en la Crónica mexicáyotl y señaló la posible referencia del documento o el informante. Por otra parte, comparó la Crónica mexicana y la Historia de las indias frase por frase para reconstruir lo que pudo haber sido la Crónica $X$. Con ello, presentó los datos comunes capítulo por capítulo, seguido de un comentario sobre elementos que deben añadirse, como las palabras en náhuatl de Tezozomoc. Como primera conclusión, Peperstraete apuntó que la Crónica mexicáyotl estuvo compuesta por tres fuentes unidas entre sí: la historia del mestizo Alonso Franco (basada en el grupo de la Tira de Peregrinación), un extracto de la Crónica $X$ y uno o más documentos genealógicos, además de estar acompañadas probablemente de explicaciones orales y en algunas ocasiones descripciones improvisadas a partir de los dibujos que tenía el autor. La conclusión final es que la Crónica mexicáyotl fue fabricada por la unión de múltiples fuentes o 
extractos de fuentes y que, a pesar de que Tezozomoc se mantuvo cercano a sus textos principales, fue rara la ocasión en que realizó una copia exacta.

El estudioso más reciente de estos temas, Gabriel Kenrick Kruell, hizo un señalamiento importante al afirmar que la parte de la Crónica mexicáyotl atribuida a Hernando Alvarado Tezozomoc, la misma que relata la migración de los mexicas, la fundación de Tenochtitlan y la etapa de su primer gobernante Acamapichtli, es un fragmento de la Crónica $X$. La razón de ello es que tales pasajes son los que más se aproximan a la versión de Durán y su Historia y a la del propio Tezozomoc en la Crónica mexicana (Kenrick Kruell 2013b: 200). Kruell parece ser partidario de la opinión de Romero Galván de que el autor de la Crónica $X$ pudo haber sido Tezozomoc, pues acepta que esta obra haya sido escrita antes de 1581 (dado que en ese año Durán la tomó como fuente para el primer volumen de su Historia de las Indias). En otros puntos, Kruell va aun más allá. Sugirió que la Crónica mexicana es la versión castellana de la Crónica $X$, y que dicha traducción, anónima, se realizó en los últimos años de vida de Tezozomoc o después de la muerte de éste. Por otro lado, también afirmó que en 1609 Tezozomoc escribió la Crónica mexicáyotl mediante la simple operación de transcribir el relato de la Crónica $X$ y añadirle un proemio. Y que (con posterioridad a esta fecha) a este producto Chimalpain le insertó otros textos, ciertos comentarios y una genealogía (Kenrick Kruell 2013b: 217-218). El sugerente trabajo de investigación y la formulación de propuestas que ha realizado Kruell no constituye retos espinosos, sino nuevas y estimulantes perspectivas con las que abordar el análisis de la historiografía de tradición indígena. En la línea de Peperstraeste, Kruell publicó en 2013 un trabajo filológico sobre el texto de la Crónica mexicáyotl, con el objetivo de reconstruir un fragmento original náhuatl de la Crónica $X$ (2013a: 301-461). Su metodología consistió en tomar las partes del texto que, a consideración del autor, fueron escritas sólo por Tezozomoc, y dejar a un lado las anotaciones adicionales de Chimalpain. El texto que eligió para iniciar su reconstrucción es un interesante prólogo, probablemente escrito por Tezozomoc en 1609, es decir, ya al final de su vida, pues no aparece en versiones anteriores de la Crónica mexicáyotl, que fueron escritas durante el siglo XVI, y no tiene paralelo ni en la Crónica mexicana, ni en la Historia de las Indias. Para elaborar su análisis, Kruell fragmentó escrupulosamente los párrafos; les dio un orden y cuando lo creyó necesario agregó o suprimió palabras, oraciones o personajes históricos de la Crónica mexicáyotl. El primer producto de su trabajo fue la reconstrucción de los tres primeros capítulos de la Crónica mexicáyotl. Por otra parte, Kruell presentó un cotejo entre el texto de la Crónica mexicáyotl y los textos de la Crónica mexicana y de la Historia de las Indias, tanto el texto náhuatl como la traducción del autor, y copió todas las palabras nahuas presentadas en el texto para poder evidenciar las equivalencias que hay entre estas tres obras, y por tanto demostrar que una versión de la Crónica mexicáyotl anterior a la copiada por Chimalpain dio origen a la Crónica mexicana y a la Historia de las Indias. Finalmente, anexó el texto reconstruido, con lo que dio un paso adelante para conocer y, como él bien dice, resucitar la Crónica X.

\section{Notas FinALES}

La apretada síntesis historiográfica que he presentado hasta aquí es lo que tenemos hoy día respecto de la identidad de la Crónica X. Más allá de los aciertos y los errores, de las quimeras y las presunciones sólidas, considero que ha sido indispensable abrir esta senda a fin de despejar el camino a las investigaciones presentes y futuras sobre la materia. En mucho porque los estudios producidos durante el siglo XX y lo que va del XXI han subrayado los problemas heurísticos y hermenéuticos con los que todavía debemos lidiar para la posible reconstrucción de ese enigmático manuscrito. En adelante, y además de detectar otras potenciales dificultades, nos queda hacer frente a los aspectos ya señalados pero aún no resueltos, por ejemplo, las evidentes similitudes estructurales de las obras que podrían tener un abrevadero común en la Crónica $X$. Otro de ellos es la fecha de su elaboración. Algo que quizá pueda emprenderse a través de un recurso aún no intentado: la comparación de la Crónica mexicana con los Anales de Tlatelolco, puesto que ya se han realizado estudios comparativos entre las obras de Durán y Tezozomoc. 
Por mi parte, coincido con Peperstrate y Kruell en cuanto a la imperiosa necesidad de realizar un estudio profundo de la Crónica mexicayotl, toda vez que ha sido identificada como la fuente más cercana a la Crónica $X$. Aunque mi propuesta es que se analice exhaustivamente por si y en si misma y no comparativamente como se ha hecho. En las siete décadas transcurridas desde la hipótesis del investigador norteamericano Robert Barlow, diversos especialistas mexicanos y extranjeros han hecho sus propios planteos y aportaciones a esa idea seminal y los han difundido en libros, artículos y tesis doctorales. En noviembre del 2015 se llevó a cabo en el Instituto de Investigaciones Históricas de la UNAM el Coloquio Internacional en Homenaje a Robert Barlow. La Crónica X setenta años después; en este marco se discutieron y propusieron otras potenciales dificultades que plantean aspectos conocidos pero aún no resueltos, por ejemplo, las evidentes hermandades estructurales de las obras que podrían tener un denominador común en la Crónica $X$. Como quiera que sea, ésta constituye aún uno de los mayores problemas heurísticos e interpretativos de la historiografía novohispana de tradición indígena. Es indiscutible que los sugerentes trabajos de investigación que han realizado y las propuestas que se han promovido, más que constituir problemas espinosos, vienen a ser nuevos y estimulantes desafíos que abordar en el análisis.

Finalmente, me permito reflexionar en voz alta: ¿por qué no atrevernos a pensar que jamás existió la Crónica $X$ ? ¿Por qué no partir del supuesto de que Tovar, Durán y Tezozomoc se basaron en una multiplicidad de textos y que sus evidentes coincidencias arraigan en la prevalencia de una historia "oficial", perfectamente conocida por sus informantes? En este sentido, muy útil resultaría ampliar la búsqueda de otros documentos con rasgos cercanos a los de la Crónica $X$, como plantean Castañeda y Oudjik. Esto apuntalaría la labor pendiente de restaurar y definir el verdadero carácter de lo que pudo ser esa fuente “común". En acatamiento a la contundente afirmación de Robert Barlow de que "Si la Crónica X no existiera, sería necesario inventarla”, me pregunto: ¿por qué no empezar desde ahora a reconstruirla en su totalidad?

\section{BIBLIOGRAFÍA}

Barlow, Robert (1990). “La 'Crónica X’: versiones coloniales de la historia de los mexica-tenochca”, en Jesús MonjarásRuiz, Elena Limón y María de la Cruz Paillés (eds.), Obras de Robert H. Barlow. Los mexicas y la Triple Alianza, v. 3, México, Instituto Nacional de Antropología, Universidad de las Américas.

Bernal, Ignacio (1947). “Los calendarios de Durán. Más confusiones alrededor de la Crónica X”, en Revista Mexicana de Estudios Antropológicos, vol. 9, pp. 125-134.

Brennan, Sallie Craven (1988). Cosmogonic use of time and space in historical narrative: The case of the "Crónica Mexicayotl". Tesis de Doctorado, Ann Arbor, Michigan, University Microfilms International.

Castañeda, María (2008). "Los anales del Grupo de la Tira de la Peregrinación o Códice X. Copias, duplicaciones y su uso por parte de los cronistas”, en Tlalocan, vol. XV, pp. 183-214.

Chavero, Alfredo (1987). “Códice Ramírez - Durán - Acosta - Tezozómoc”, en Hernando Alvarado Tezozomoc, Crónica mexicana, Edición facsimilar de la de 1878, anotada por Manuel Orozco y Berra, precedida del Códice Ramírez, $4^{a}$ ed., México, Editorial Porrúa.

Colston, Stephen A. (1973). Fray Diego Duran's historia de las Indias de Nueva España e Islas de la Tierra Firme: a historiographical analysis. Tesis de Doctorado, Philosophy in History, Los Angeles, University of California, University Microfilms International.

Colston, Stephen A. (1977). “A comment on dating the 'Crónica X'”, en Tlalocan (7), pp.371-377.

Cortés Campos, Rocío Leticia (1998). Estrategias narrativas en el discurso en la crónica mexicana y la crónica mexicáyotl de Hernando Alvarado Tezozomoc. Tesis de Doctorado, Philosophy in Spanish, University of WisconsinMadison.

Graham Classen, Ann Marie (1998). Dos interpretaciones de la historia de los mexicas: un análisis comparativo de la Crónica mexicana de Hernando Alvarado Tezozómoc y la Historia de las Indias de Nueva España e islas de Tierra 
Firme de fray Diego Durán. Tesis de Doctorado, México, Universidad Nacional Autónoma de México, Facultad de Filosofía y Letras.

Kenrick Kruell, Gabriel (2013a). "Resucitando la Crónica X reconstrucción filológica de un fragmento inicial de la crónica mexicáyotl de Hernando de Alvarado Tezozómoc”, en Tlalocan (19), pp. 301-461.

Kenrick Kruell, Gabriel (2013b). "La Crónica mexicáyotl: versiones coloniales de una tradición histórica mexica tenochca”, en Estudios de Cultura Nábuatl, vol. 45, pp. 198-232.

Kenrick Kruell, Gabriel (2013c). "Reseña bibliográfica de La 'Chronique X': Reconstitution et analyse d'une source perdue fondamentale sur la civilisation Aztèque, d'après l'Historia de las Indias de la Nueva España de D. Durán (1581) et la Crónica Mexicana de F. A. Tezozomoc (ca.1598)", en Estudios de Cultura Nábuatl, vol. 46, pp. 320-327.

Kirchhoff, Paul (1951). "El autor de la segunda parte de la Crónica Mexicáyotl", en Homenaje al doctor Alfonso Caso, México, Imprenta Nuevo Mundo.

Oudijk, Michel R. y María Castañeda de la Paz (2009). "El uso de fuentes históricas en pleitos de tierras: la Crónica X y la Ordenanza de Cuauhtémoc", en Tlalocan, vol. XVI, pp. 255-278.

Peperstraete, Sylvie (2007). La "Chronique X": Reconstitution et analyse d'une source perdue fondamentale sur la civilisation Aztèque, d'après l'Historia de las Indias de la Nueva España de D. Durán (1581) et la Crónica Mexicana de F. A. Tezozomoc (ca.1598), Oxford, Archeopress.

Peperstraete, Sylvie (2010). "Nouvelles hypothèses sur la Crónica mexicáyotl”, en Journal de la société des américanistes, pp.7-37, doi: http://jsa.revues.org/11247.

Ramírez, Fernando (1987). "Códice Ramírez. Advertencia”, en Hernando Alvarado Tezozomoc, Crónica mexicana, Edición facsimilar de la de 1878, anotada por Manuel Orozco y Berra, precedida del Códice Ramírez, 4a ed., México, Porrúa.

Romero Galván, José Rubén (2003). “La Crónica X”, en José Rubén Romero Galván (coord.), Historiografia mexicana de tradición indigena, México, Universidad Nacional Autónoma de México.

Tena, Rafael (1997). “Revisión de la hipótesis sobre la Crónica X”, en Constanza Vega Sosa, Salvador Rueda Smithers y Rodrigo Martínez Baracs (eds.), Códices y documentos sobre México. Segundo Simposio, pp.163-178,México, Instituto Nacional de Antropología e Historia, Consejo Nacional para la Cultura y las Artes.

\section{Notas}

1 Este texto es producto de mi proyecto de investigación radicado en la Dirección de Estudios Históricos-INAH (Instituto Nacional de Antropología e Historia, México), titulado "Crónicas novohispanas y andinas, siglos XVI-XVII".

2 El "manuscrito Tovar" se encuentra en la Biblioteca John Carter Brown, cuya elaboración fue realizada por el sacerdote Juan de Tovar de la Compañía de Jesús para 1584. José Fernando Ramírez encontró una copia incompleta de ese documento en la desaparecida biblioteca del Convento Grande de San Francisco de México, durante su demolición en 1856, copia a la que convencionalmente se le ha llamado "códice Ramírez" y que también data del siglo XVI. José de Acosta también es un sacerdote jesuita que participó de la organización eclesiástica en los territorios americanos de la Corona española durante la segunda mitad del siglo XVI, sobre todo durante la conformación del Virreinato del Alto Perú. Hacia 1586 viajó a la Nueva España, y ahí terminó de formar su obra Historia natural y moral de las indias que se publicaría en la península ibérica, geografía en la que falleció siendo rector de la Universidad de Salamanca en 1600. Fray Diego Durán perteneció a la Orden de Predicadores de Santo Domingo de Guzmán, y su obra Historia de las Indias de Nueva España e Islas de Tierra Firme fue realizada en la Nueva España cerca de 1579. Por último, Hernando Alvarado Tezozomoc es descendiente de Don Diego Huanitzin, gobernador de Tenochtitlan, lo que le permitió posicionarse entre el conocimiento del estrato social descendiente de los antiguos gobernantes prehispánicos del Altiplano Central Mesoamericano, posición indispensable para entender la escritura de su Cronica mexicana, así como de la Crónica mexicayotl, texto en el que también se ve involucrado otro cronista indígena de nombre Domingo de San Antón Muñón Chimalpahin Cuauhtlehuanitzin.

3 La presencia de los europeos y, sobre todo, la imposición de nuevas formas culturales, trajeron como consecuencia obligada que otras maneras de registrar el pasado irrumpieran en el universo de las historias producidas y trasmitidas entre los indígenas novohispanos. Es irrefutable que en las historias que escribieron los indígenas en esa segunda mitad del siglo se perciben con claridad esquemas de explicación histórica que corresponden a la tradición europea, en torno 
a los cuales fue ordenada información de gran riqueza proveniente de las fuentes de la más pura estirpe prehispánica. Así se percibe en el contenido de códices pictográficos, en el de los códices glosados e incluso en la información trasmitida a través de la oralidad, es decir, los testimonios que habían recibido de boca de sus parientes y mayores, quienes formaban parte de la antigua nobleza y habían sido testigos de los hechos que narraban. Véase a José Rubén Romero Galván "Introducción", Historiografía novohispana de tradición indigena, en Juan A. Ortega y Rosa Camelo (coords.), Historiografía mexicana, vol. I, Ciudad de México, Universidad Nacional Autónoma de México - Instituto de Investigaciones Históricas (UNAM-IIH), 2003.

4 Este artículo fue publicado originalmente en la Revista de Estudios Antropológicos en 1945 y se sustentó en la conferencia dictada por Barlow en la Facultad de Filosofía y Letras de la UNAM el mismo año.

5 Hasta fecha muy reciente la obra denominada Crónica mexicáyotl había sido atribuida al cronista Hernando Alvarado Tezozómoc. Sin embargo, a principios de los ochenta del siglo XX aparecieron en la Biblioteca de la Sociedad Bíblica de Londres tres volúmenes de la colección que en el siglo XVII perteneció a don Carlos Sigüenza y Góngora. Entre otros manuscritos autógrafos de Chimalpahin, uno de tales volúmenes contenía la referida Crónica mexicáyotl. A través del análisis de dicho texto, hoy se ha podido determinar que la mayor parte de esta historia fue, en realidad, obra del cronista chalca, quien, por propia mano, agregó dos fragmentos, uno escrito originalmente por Alvarado Tezozómoc y el otro por un mestizo de nombre Alonso Franco. 